\title{
Neuromarketing: Neural Explanations for Consumer Behaviours
}

\author{
Sachin Mishra*1 and Sunil Kumar Mishra ${ }^{2}$ \\ ${ }^{1}$ Cognitive Neuroimagine Centre, Nanyang Technological University, 59 Nanyang Drive, Singapore \\ ${ }^{2}$ Patronage Institute of Management Studies, India
}

*Corresponding author: Sachin Mishra, Cognitive Neuroimagine Centre, Nanyang Technological University, 59 Nanyang Drive, Singapore

Sunil Kumar Mishra, Patronage Institute of Management Studies, Uttar Pradesh, India

\begin{tabular}{|c|c|}
\hline ARTICLE INFO & ABSTRACT \\
\hline
\end{tabular}

\section{Introduction}

\section{The Synergy of Neuroimaging and Marketing Principles}

The advents of neuroimaging techniques and modalities have shown immense potential in various fields of research. Imaging techniques are capable of identifying and mapping different regions of the brain in relation to their functions, which acts as a key to various research related to cognition and neurophysiology. In addition to this, a more comprehensive insight of human brain can be achieved by blending the anatomical and functional perspectives through hybridization. With consistently improving technologies, there have been many developments in fused imaging techniques over the past few decades. The global market of consumer products and services is a potential hotspot for researchers to demonstrate the applications of neuroimaging and brain mapping. Neuromarketing principles applies neuropsychology to marketing research and study the subject's response to marketing stimuli in terms of cognitive response with brain signals [1]. In comparison with traditional research methods, neuromarketing is proving itself to be advanced, emerging and advantageous, as a marketing research tool.

\section{Neuroimaging Modalities: Delving Thoughts of Consum- ers}

Vibrant tools are available which applies neuroscience data for consumer research and further provide neural explanations for consumer behaviours. Counting from the simple and peripheral non-neuroimaging Electrooculography (EOG) to advanced neuro imaging hybrid modalities like EEG-fMRI, MEG-MRI etc. [2] have potentials to be used for neuromarketing. Even eye movement data from EOG or eye-tracking camera can provide data for qualitative modelling of the decision-making process [3]. Researchers believe that most consumers' decisions are made in a split second, and many of those decisions are made subconsciously. EEG and MEG can measure electrical (magnetic field in case of MEG) activity in the brain which is associated with increased or reduced focus and / or excitement [4,5]. Portable and sophisticated sensors like EEG and fNIRS can measure consumer response in a real and appropriate environment such as mall, retail stores, showrooms etc., instead of simulated scenarios of virtual reality and computer images [6]. In case of fMRI, though not convenient in portable research, offers cutting edge methods that can delve into subconscious processes which has a potential to significantly improve the effectiveness of advertising in real market. Various advanced and hybrid imaging techniques are being explored for potential applications in market research.

\section{Various Neuroimaging Approaches in Action}

Consumer neuroscience amalgamates consumer research with advanced neuroscience approaches and protocols. The goal of these approaches is to find neural explanations for consumer behaviours, their choices and decision making. The objectives of major market research paradigms revolve around product, advertising, branding and pricing. In neuroscience paradigms, these can be 
assessed by decision making, reward, memory and emotions. Nonneuroscience-based approaches are

a) Facial coding, connects emotions to body language, specifically facial expressions.

b) Eye tracking, to monitor attention and focus in advertisement.

c) Galvanic Skin Response, responding to interaction between external events and the physical psychological state.

Neuroscience based approaches differs with modalities and have their own advantages and disadvantages over each other. EEG and MEG based approaches are mostly similar, with difference in their way of stimulus delivery and response measurement. Cortical activations associated to the decisions of an experimental subject is the basic approach which is generally followed [7]. Beside this, the average absolute and relative power spectra calculation in different frequency band also present an overview of psychological state of a person. Combined with machine learning and various data processing tools, these data can provide robust results. On the other hand, fMRI works with the neural substrate theories of consumer behaviour for some research models [8]. In other models, localisation and activation based resting-state and task study is performed to get functional images, which can be analysed further. The evolution of new modalities and approaches of data processing are opening new gateways in neuromarketing.

\section{Conclusion and Future Scope}

There are various approaches available with neuroimaging modalities and many new approaches are in process of development, which when combined with artificial intelligence approaches, can give a better picture to marketing scientist and product developers about choices and behaviours of consumers. The limitations of the existing approaches lie in the accuracy and universalism of the attained result, as behaviour and perceptions show plasticity and variation, both, in same person with time and location, and person to person. Beside this, opinion on responsible dual use are required to be produced by controlling authorities to prevent exploitation of consumers and their data. However, the prediction results with present approaches are promising and the researched frameworks can be used for better business models.

\section{References}

1. Lee N, Broderick AJ, Chamberlain L (2007) What is 'neuromarketing'? A discussion and agenda for future research. International journal of psychophysiology 63(2): 199-204.

2. Padmanabhan P, Nedumaran AM, Mishra S, Pandarinathan G, Archunan G, et al. (2017) The Advents of Hybrid Imaging Modalities: A New Era in Neuroimaging Applications. Advanced Biosystems 1(8): 1700019.

3. Marandi RZ, Sabzpoushan SH (2015) Qualitative modeling of the decision-making process using electrooculography. Behavior research methods 47(4): 1404-1412.

4. Yadava M, Kumar P, Saini R, Roy PP, Dogra DP (2017) Analysis of EEG signals and its application to neuromarketing. Multimedia Tools and Applications 76(18): 19087-19111.

5. Vecchiato G, Astolfi L, De Vico Fallani F, Toppi J, Aloise F, et al. (2011) On the use of EEG or MEG brain imaging tools in neuromarketing research. Computational intelligence and neuroscience.

6. Ogino M, Mitsukura Y (2018) A Mobile Application for Estimating Emotional Valence Using a Single-Channel EEG Device. In 2018 57 Annual Conference of the Society of Instrument and Control Engineers of Japan (SICE), pp. 1043-1048.

7. Braeutigam S (2005) Neuroeconomics-From neural systems to economic behaviour. Brain research bulletin 67(5): 355-360.

8. Hsu MYT (2018) Cognitive systems research for neuromarketing assessment on evaluating consumer learning theory with fMRI: Comparing how two Word-of-Mouth strategies affect the human brain differently after a product harm crisis. Cognitive Systems Research 49: 49-64.

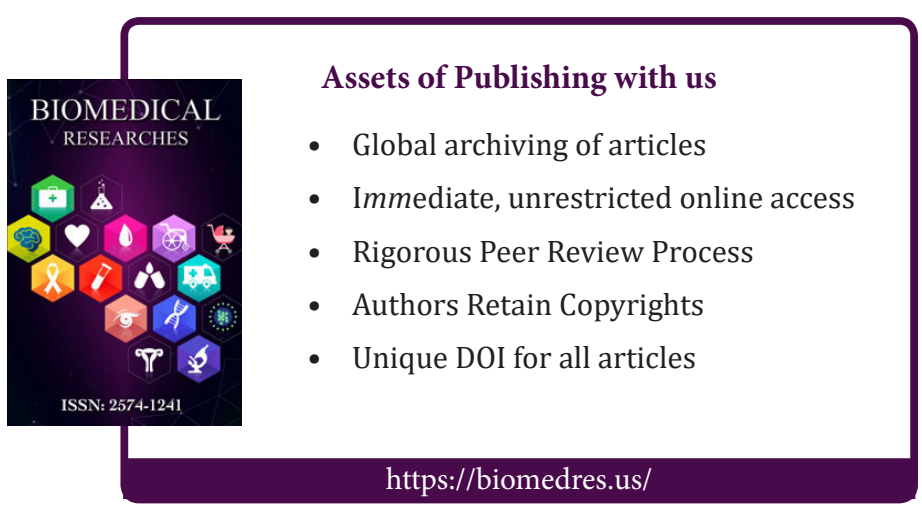

\section{ISSN: 2574-1241}

DOI: 10.26717/BJSTR.2019.16.002792

Sachin Mishra. Biomed J Sci \& Tech Res

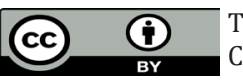

This work is licensed under Creative Commons Attribution 4.0 License

Submission Link: https://biomedres.us/submit-manuscript.php 